\title{
Comparative thermodynamic analysis of cyclic nucleotide binding to protein kinase $A$
}

\section{Daniela Moll', Sonja Schweinsberg', Christian Hammann ${ }^{2}$ and Friedrich W. Herberg ${ }^{1, *}$}

${ }^{1}$ Department of Biochemistry, University of Kassel, Heinrich-Plett-Str. 40, D-34132 Kassel, Germany ${ }^{2}$ Research Group Molecular Interactions, Department of Genetics, University of Kassel, Heinrich-Plett-Str. 40, D-34132 Kassel, Germany

*Corresponding author

e-mail: herberg@uni-kassel.de

\begin{abstract}
We have investigated the thermodynamic parameters and binding of a regulatory subunit of cAMP-dependent protein kinase $(\mathrm{PKA})$ to its natural low-molecular-weight ligand, CAMP, and analogues thereof. For analysis of this model system, we compared side-by-side isothermal titration calorimetry (ITC) with surface plasmon resonance (SPR). Both ITC and SPR analyses revealed that binding of the protein to CAMP or its analogues was enthalpically driven and characterised by similar free energy values $\left(\Delta G=-9.4\right.$ to $\left.-10.7 \mathrm{kcal} \mathrm{mol}^{-1}\right)$ for all interactions. Despite the similar affinities, binding of the cyclic nucleotides used here was characterised by significant differences in the contribution of entropy $(-T \Delta S)$ and enthalpy $(\Delta H)$ to $\Delta G$. The comparison of ITC and SPR data for one CAMP analogue further revealed deviations caused by the method. These equilibrium parameters could be complemented by thermodynamic data of the transition state $\left(\Delta H^{\neq}, \Delta G^{\neq}, \Delta S^{\neq}\right)$for both association and dissociation measured by SPR. This direct comparison of ITC and SPR highlights method-specific advantages and drawbacks for thermodynamic analyses of protein/ligand interactions.
\end{abstract}

Keywords: cAMP-dependent protein kinase; isothermal titration calorimetry; protein-ligand interaction; surface plasmon resonance.

\section{Introduction}

cAMP-dependent protein kinase (PKA) represents a paradigm for the whole protein kinase family (Manning et al., 2002). In its inactive state, PKA consists of two dimeric regulatory $(R)$ subunits and two catalytic $(C)$ subunits. Activation of PKA occurs upon binding of the second messenger CAMP to the two highly conserved cyclic nucleotide-binding domains of the $\mathrm{R}$ subunit (Berman et al., 2005; see Figure 1). The binding of cAMP is a key event in signal transduction regulated by PKA. The lowmolecular-weight ligand CAMP and analogues thereof are the point of action of drugs against diseases in which PKA involvement has been shown, such as breast cancer
(Stratakis and Cho-Chung, 2002) and human immunodeficiency caused by HIV (Aandahl et al., 1998; Rahmouni et al., 2001). The Carney complex disease (CNC) (Stergiopoulos and Stratakis, 2003) and polycystic ovary syndrome (PCOS) (Ek et al., 2002) are PKA-related diseases and are also prospective fields for drug development.

PKA is among the best-characterised protein kinases and thus serves as a prototype of this important family of enzymes. Several high resolution crystal structures are available for monomeric $R$ subunits with (Su et al., 1995) and without (Wu et al., 2004a) cAMP, or analogues thereof (Wu et al., 2004b). Recently, the structure of a truncated $R$ subunit in complex with the $C$ subunit of PKA was also obtained (Kim et al., 2005). These structures are complemented by a vast set of equilibrium data from kinetic studies, describing the binding of cAMP analogues to the regulatory subunit (Schwede et al., 2000a). A combination of structural and kinetic data can lay the basis for the rational design of new CAMP analogues in drug development (Schwede et al., 2000b). Despite substantial progress in this field, it is unknown why PKA antagonists work under certain conditions or how they could be optimised for improved efficacy. This gap could be filled by determining missing thermodynamic information, which could provide an insight into the structurefunction relationship and thus yield valuable information on affinity and the mechanism of action.

The thermodynamic parameters $\Delta G$ (free energy), $\Delta H$ (enthalpy) and $\Delta S$ (entropy) are related to each other and to the equilibrium binding constant $\left(K_{\mathrm{d}}\right)$ according to:

$\Delta G^{\circ}=\Delta H^{\circ}-T \Delta S^{\circ}=R T n K_{\mathrm{d}}$,

where $T$ is the absolute temperature and $R$ is the universal gas constant.

Direct measurements of thermodynamic parameters are usually carried out by isothermal titration calorimetry (ITC), a method that directly detects the heat generated or absorbed during the binding event. Since only a small subset of interactions take place athermally, i.e., without heat changes, ITC is considered a universal method. This homogeneous assay has no molecular weight limitations and is also absolutely label-free; however, it requires a substantial amount of the interaction partners.

An alternative approach is the indirect determination of thermodynamic data from kinetic measurement of association and dissociation. Usually, individual experiments require a smaller number of binding partners than ITC. However, experiments need to be carried out at variable temperatures, which is eventually time-consuming and costly in terms of materials. Surface plasmon resonance (SPR) can be used to determine highly accurate kinetic rate constants. However, under certain circumstances, apparent rate constants may not reflect the biological 


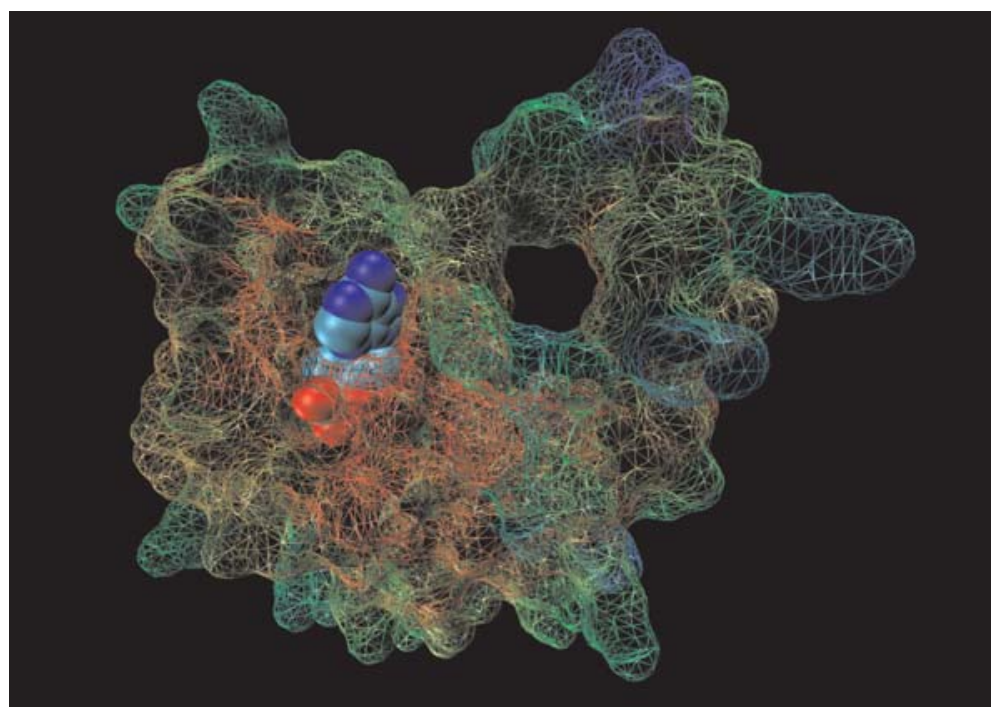

Figure 1 Wireframe representation of one of the two evolutionarily highly conserved cyclic nucleotide binding domains of the regulatory subunit of PKA (bRl $\alpha$ 113-260; PDB no. 1RGS) with bound cAMP.

The Figure was created using standard settings in VMD 1.8.4.

system correctly due to the immobilisation process itself, mass transfer limitations or rebinding effects (Herberg and Zimmermann, 1999).

Here, we compare the suitability of ITC and SPR for the determination of thermodynamic parameters. As a model system, we used the well-studied interaction of two cAMP analogues to a truncated $R$ subunit of PKA.

\section{Results}

To determine thermodynamic parameters from SPR data, we used the CAMP binding domain $A$ of the bovine $R$ subunit la of PKA (bRl $\alpha$ 92-260), which still retains the ability to interact with the $\mathrm{C}$ subunit (Ringheim and Taylor, 1990). Compared to the full-length, multi-domain PKA R subunit with four cAMP binding sites, data analysis for this construct is greatly facilitated, because a 1:1 stoichiometry is expected for $\mathrm{R}$ subunit/cAMP binding (Ringheim and Taylor, 1990). This construct was previously used to elucidate the activation mechanism of PKA (Hahnefeld et al., 2005) and is kinetically (Ringheim and Taylor, 1990) and structurally (Anand et al., 2003; Kim et al., 2005) well characterised. In this study, we used the physiological ligand CAMP and two of its analogues, 6-AHCAMP and 8-AHA-CAMP (Figure 2). Equilibrium data for 8-AHA-cAMP binding to PKA $R$ subunit are identical to those for CAMP (Ogreid et al., 1989; Schwede et al., 2000a), while 6-AH-cAMP binding is slightly stronger (Moll et al., 2006). Both analogues can be coupled to sensor surfaces via the primary amino group of their linker, thus allowing for solid-phase analyses (Hahnefeld et al., 2004; Gesellchen et al., 2005), and can also be used in homogeneous assay formats such as ITC.

\section{ITC, the classical tool for thermodynamic analysis}

We used ITC (Wiseman et al., 1989) to directly determine the thermodynamic parameters $(\Delta G, \Delta H$ and $\Delta S)$ of the<smiles>Nc1ncnc2c1ncn2[C@@H]1COP(=O)(O)OC[C@@H]1O</smiles>

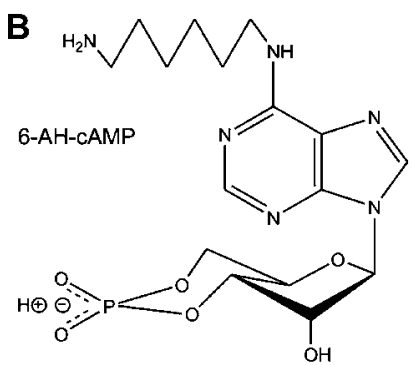

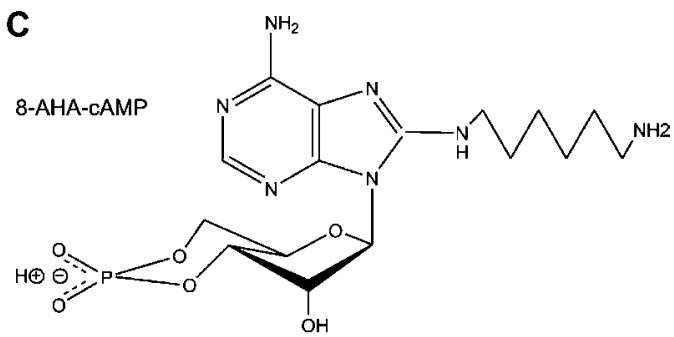

Figure 2 Structural formula of the cyclic nucleotides used in this study.

(A) The natural PKA ligand cAMP. (B) 6-AH-cAMP featuring an aminohexyl linker at the exocyclic amino group (N6) of cAMP. (C) 8-AHA-CAMP with an (6-aminohexyl)amino linker at C8 of CAMP.

interaction of the PKA R subunit with CAMP (and analogues thereof). In these experiments, aliquots of the lowmolecular-weight binding partner were injected into a concentrated protein solution of bRl $\alpha$ 92-260 in the cal- 
orimeter sample cell. Upon each injection, accompanying heat changes were measured as a function of time. Detailed descriptions of the method and background can be found in Ladbury and Chowdhry (1996). Titration of each of the three ligands to the protein resulted in exothermic binding (Figure 3, upper panels). Areas under the injection peaks were integrated, corrected for dilution and plotted versus the protein/nucleotide molar ratio (Figure 3, lower panels). We fitted the data to a 'one set of sides' model using MicroCal Origin software. These calculated curves describe the experimental data very well, as shown by the fits in the lower panels of Figure 3. Using data from heat measurements (corresponding to $\Delta H$ ) and $K_{\mathrm{d}}$ from the fits, $\Delta G$ and $\Delta S$ were calculated according to Eq. (1). The resulting thermodynamic parameters for the interaction of each of the ligands with $b R l \alpha$ 92-260 are summarised in Table 1.

The interaction of unmodified cAMP with this PKA R subunit is dominated by enthalpic contributions $(\Delta H=$ $-14.8 \pm 0.3 \mathrm{kcal} \mathrm{mol}^{-1}$ ). An opposite entropic term reduces the free energy $\Delta G$ to $-10.0 \pm 0.1 \mathrm{kcal} \mathrm{mol}^{-1}$, which corresponds to $K_{\mathrm{d}}$ of $38 \pm 6 \mathrm{nM}$.

The analogue 6-AH-cAMP ( $\left.\Delta G=-10.7 \pm 0.1 \mathrm{kcal} \mathrm{mol}^{-1}\right)$ binding to the PKA R subunit is slightly stronger than for cAMP. However, this small difference in affinity is made up of considerably stronger but opposite changes in the contribution of enthalpy and entropy, which largely compensate one another (Table 1). Binding affinities of 8AHA-cAMP $\left(\Delta G=-9.9 \pm 0.2 \mathrm{kcal} \mathrm{mol}^{-1}\right)$ and cAMP $(\Delta G=$ $-10.0 \pm 0.1 \mathrm{kcal} \mathrm{mol}^{-1}$ ) to the $\mathrm{R}$ subunit were the same within the error of the measurement. Again, these identical $\Delta G$ values were associated with significant differences in the contribution of entropy and enthalpy. For 8-AHA-cAMP binding, both $\Delta H$ and $\Delta S$ were reduced by
Table 1 Equilibrium thermodynamic constants measured by ITC.

\begin{tabular}{lcccc}
\hline Ligand & $\begin{array}{c}K_{\mathrm{d}}{ }^{\mathrm{a}} \\
(\mathrm{nM})\end{array}$ & $\begin{array}{c}\Delta \mathrm{G}^{\mathrm{a}} \\
\left(\mathrm{kcal} \mathrm{mol}^{-1}\right)\end{array}$ & $\begin{array}{c}\Delta H \\
\left(\mathrm{kcal} \mathrm{mol}^{-1}\right)\end{array}$ & $\begin{array}{c}\Delta S \\
(\mathrm{cal} \mathrm{mol}-1 \\
\left.\mathrm{K}^{-1}\right)\end{array}$ \\
\hline CAMP & $38 \pm 6$ & $-10.0 \pm 0.1$ & $-14.8 \pm 0.3$ & $-16 \pm 1$ \\
6-AH-cAMP & $10 \pm 1$ & $-10.7 \pm 0.1$ & $-16.3 \pm 0.1$ & $-19 \pm 1$ \\
8-AHA-cAMP & $46 \pm 15$ & $-9.9 \pm 0.2$ & $-12.6 \pm 0.5$ & $-11 \pm 1$ \\
\hline
\end{tabular}

a Determined at $293 \mathrm{~K}$.

the same amount compared to cAMP, which results in identical $\Delta G$ values for both ligands (Table 1).

Thus, all three nucleotides bind to the PKA R subunit in an enthalpically driven way. ITC allows the enthalpy and entropy contributions to the affinities to be distinguished, revealing significant differences in the binding mode of protein/ligand interactions.

\section{Determination of kinetic data from SPR}

Binding affinities can be determined using SPR analyses from real-time kinetic data (Karlsson and Falt, 1997). This widely applied method allows separate determination of association and dissociation rate constants $\left(k_{\text {ass }}\right.$ and $\left.k_{\text {diss }}\right)$, from which the equilibrium dissociation constant $\left(K_{\mathrm{d}}\right)$ for a given interaction can be calculated (Karlsson and Falt, 1997). For fully automated determination of kinetic rate constants, we used a Biacore T100 system. From these kinetic data, thermodynamic parameters for the equilibrium and the transition state can be calculated from a single experimental set-up.

SPR is used to detect mass changes upon complex formation; thus, the smaller of the interaction partners is
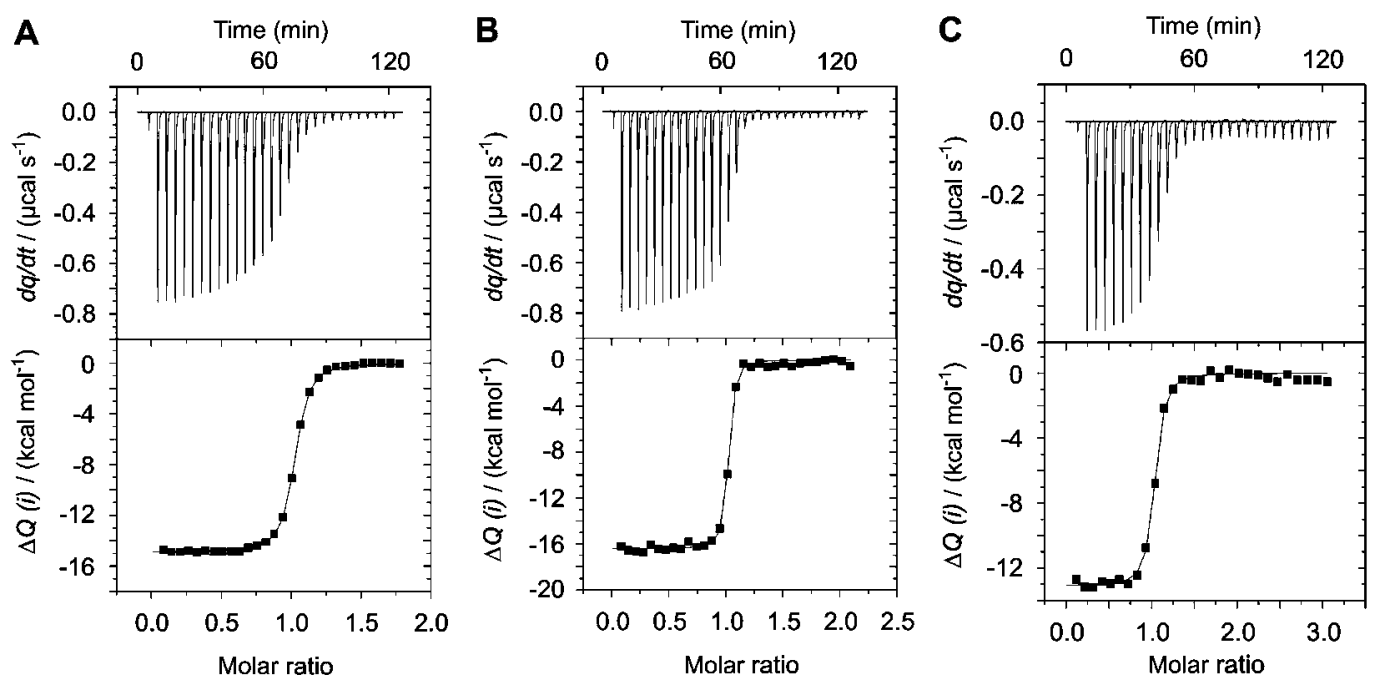

Figure 3 Binding isotherms derived from isothermal titration calorimetry (ITC) for the interaction of the bovine PKA R subunit bRl $\alpha$ 92-260 and the three nucleotides.

In each series, the ligand was injected at $293 \mathrm{~K}$ into $10 \mu \mathrm{M}$ protein, dialysed against buffer A supplemented with 1 mM $\beta$-mercaptoethanol. Ligands were dissolved in dialysis buffer to minimise differences in buffer composition. For each analogue, the heat released upon each injection (dq/dt) was measured as a function of time (upper panels). Integrated areas under the injection peaks were plotted as heat exchange per mol of injectant $[\Delta Q(i)]$ relative to the molar ratio of protein to ligand (lower panels). Curves in the lower panels were derived from best least-square fit analysis, applying a model with one binding site (performed with MicroCal Origin software). Thermodynamic data derived from these analyses are summarised in Table 1 . (A) Injection of $5-\mu$ l aliquots of $271 \mu \mathrm{M}$ cAMP. (B,C) Injection of 10- $\mu$ l aliquots of $140 \mu \mathrm{M}$ 6-AH-cAMP and 8-AHA-cAMP, respectively. 
frequently immobilised on the sensor chip surface to improve the signal/noise ratio. Injection of the smaller binding partner, i.e., cAMP, onto the immobilised protein results in weak binding signals due to the small mass difference. Furthermore, for strong binding events such as PKA to cyclic nucleotides, regeneration of coupled protein on the chip surface is difficult.

Using NHS/EDC chemistry (Johnsson et al., 1991), we covalently coupled the cyclic nucleotide analogues 6$\mathrm{AH}-\mathrm{cAMP}$ and 8-AHA-cAMP via the primary amino group of their linker to the sensor chip surface and determined binding of the PKA R subunit. This set-up is not suitable for investigating interactions of PKA and unmodified cAMP, because coupling the (primary) amino group at C6 of cAMP does not result in functional surfaces (Zimmermann and Herberg, unpublished observation).

Kinetic data for interaction of the PKA $R$ subunit with 6-AH-cAMP and 8-AHA-cAMP were recorded at seven temperatures, covering a range of $32 \mathrm{~K}$ (Figure 4). In these experiments, 2.5-640 nM of the PKA R subunit was injected for $180 \mathrm{~s}$ onto the immobilised ligand and changes in the SPR signal were measured in response units $(R U)$. After this association phase, injection of buffer for a further $180 \mathrm{~s}$ allowed monitoring of dissociation by the same principle. Figure 4 shows the SPR signals detected for the different protein concentrations in red, and fits (see below) as dotted black lines. For both analogues, an increase in temperature led to faster association and dissociation rates. A Langmuir 1:1 binding model (Langmuir, 1916, 1918; reviewed in Hahnefeld et al., 2004) was applied to extract $k_{\text {ass }}$ and $k_{\text {diss }}$ values for each temperature and analogue used. The resulting fits describe the experimental data very well and were plotted in Figure 4 as dotted black lines using the Biacore T100 Evaluation Software.

\section{Calculation of equilibrium thermodynamic parameters from kinetic data}

The equilibrium dissociation constants $\left(K_{\mathrm{d}}\right)$ were determined as the ratio of $k_{\text {diss }}$ and $k_{\text {ass }}$ (derived in Figure 4). In the temperature range studied here $\left(5-37^{\circ} \mathrm{C}\right)$, the $K_{\mathrm{d}}$ for binding of the PKA $R$ subunit to both ligands increased nearly ten-fold, from 14 to $120 \mathrm{nM}$ for $6-\mathrm{AH}-\mathrm{cAMP}$ and from 35 to $290 \mathrm{~nm}$ for 8-AHA-cAMP. This is consistent with a lower equilibrium binding signal at higher temperatures (Figure 4).

The $K_{\mathrm{d}}$ values equivalent to equilibrium $\Delta G$ were used to determine equilibrium $\Delta H$ and $\Delta S$ according to Eq. (1), rearranged for use in van't Hoff plots:

$\ln K_{d}=\frac{\Delta H}{R} \frac{1}{T}-\frac{\Delta S}{R}$.

For both cAMP analogues, the van't Hoff plots (InK $K_{d}$ vs. $1 / T)$ are linear $\left(r^{2}>0.99\right)$, indicating that equilibrium enthalpy and entropy do not vary significantly in the temperature range studied (Figure 5).

The equilibrium thermodynamic parameters determined with the Biacore T100 system for the two different cAMP analogues are very similar (Table 2). Compared to data from ITC experiments (Table 1), binding of either cAMP analogue to the PKA R subunit seems to be slight- ly weaker. Both methods, however, indicate that 6-AHcAMP is the stronger binder. Overall, the thermodynamic values $(\Delta G, \Delta H$ and $\Delta S)$ for the interaction of 8-AHAcAMP determined with ITC and SPR are in very good agreement. For 6-AH-cAMP, however, the two methods reveal differences, which might be due to the coupling requirement in SPR measurements (see the discussion section).

\section{Transition-state thermodynamic parameters}

From $k_{\text {ass }}$ determined at different temperatures, the thermodynamic parameters of transition-state formation for association of the ligand and protein can be calculated according to the Eyring equation:

$\ln \frac{k_{\mathrm{ass}}}{T}=-\frac{\Delta H_{\mathrm{ass}}^{\neq}}{R} \frac{1}{T}+\ln \frac{k_{\mathrm{B}}}{h}+\frac{\Delta S_{\mathrm{ass}}^{\neq}}{R}$,

where $T$ is the absolute temperature, $k_{\text {ass }}$ is the association rate constant for the activated complex determined at temperature $T, k_{\mathrm{B}}$ is the Boltzmann constant and $h$ is the Planck constant. For $k_{\text {diss }}$, equivalent calculations reveal the thermodynamic parameters for the transition during the reverse reaction (Figure $6 \mathrm{~A}$ ). In Eyring plots (Ink/T vs. $1 / T$ ), we used the kinetic rate constants for each cAMP analogue to obtain these sets of thermodynamic parameters (Figure 6B,C). Between $5^{\circ} \mathrm{C}$ and $30^{\circ} \mathrm{C}$, these plots result in straight lines $\left(r^{2}>0.98\right)$, which indicates that the heat capacity is temperature-independent over this range. Inclusion of the $37^{\circ} \mathrm{C}$ measurements decreased the linearity of the Eyring plots significantly. Therefore, we only used data from the linear range of the Eyring plots to extract transition-state data. Enthalpies $\left(\Delta H_{\text {ass }}^{\neq}\right.$and $\left.\Delta H_{\text {diss }}^{\neq}\right)$were determined from the slope and entropies $\left(\Delta S_{\text {ass }}^{\neq}\right.$and $\left.\Delta S_{\text {diss }}^{\neq}\right)$from the intercept with the yaxis (Table 3 ) using the Biacore T100 Evaluation software.

The free energy of the transition states $\left(\Delta G_{\text {ass }}^{\neq}\right.$and $\Delta G_{\text {diss }}^{\neq}$) was determined according to Eq. (1) (Table 3). Formation of the association transition states shows nearly identical thermodynamic parameters for both cAMP analogues, and the same holds for dissociation. This indicates that the binding and unbinding processes of the ligands and the PKA R subunit proceed in a similar manner. Thus, the position at which the two cAMP analogues are coupled to the sensor surface (Figure 2) does not seem to influence protein binding in SPR measurements.

\section{Discussion}

The development of therapeutic substances has been strongly advanced by rational drug design, which involves achieving full understanding of a given biomolecular interaction by combining structural, kinetic and thermodynamic information (Doyle et al., 1996; Noble et al., 2004; Holdgate and Ward, 2005; Whitesides and Krishnamurthy, 2006). As pointed out by Ladbury (2004), the availability of thermodynamic data adds important information to the decision-making process in drug development. In particular, when compounds have similar affinities, their enthalpy $(\Delta H)$ values can be used to select one as the preferred lead compound for optimi- 
A
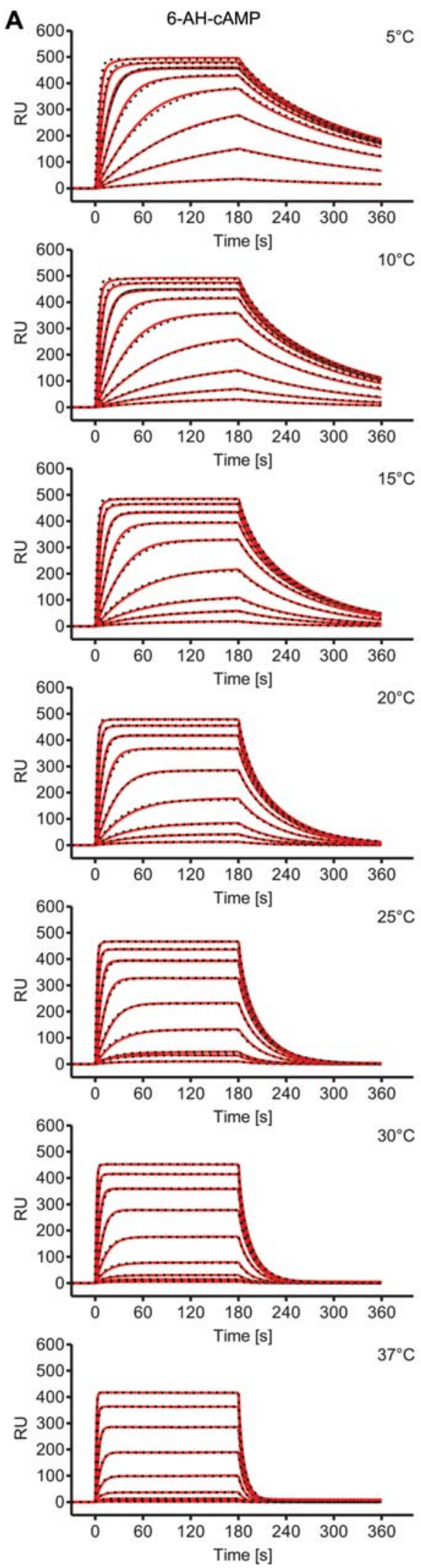

B
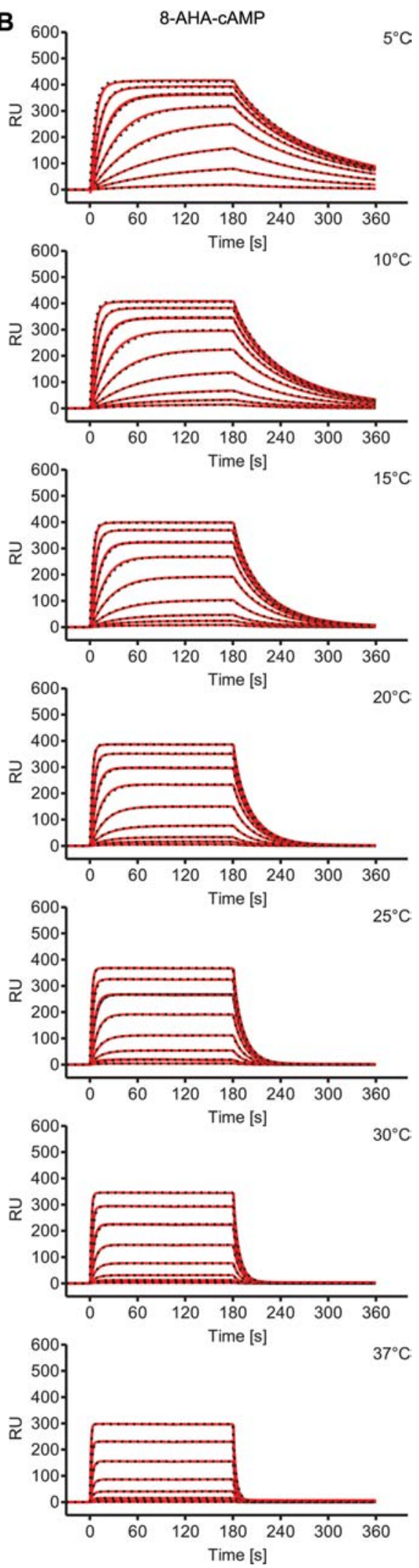

Figure 4 Surface plasmon resonance (SPR) analysis of the interaction of the bovine PKA R subunit bRl $\alpha$ 92-260 and two cAMP analogues at different temperatures using a Biacore T100 system.

6-AH-cAMP (A) or 8-AHA-cAMP (B) was immobilised on separate sensor surfaces using standard amine coupling chemistry. The PKA R subunit was diluted in buffer A supplemented with $0.005 \%(\mathrm{v} / \mathrm{v})$ surfactant $\mathrm{P} 20$, which was also used as running buffer. SPR signals in response units (RU) were detected for association (0-180 s) and dissociation (180-360 s) of bRl $\alpha$ 92-260 at the temperature indicated in each sensorgram. Each graph shows kinetic data for serial injections of eight protein concentrations, with data for the lowest $(2.5 \mathrm{~nm})$ to the highest $(640 \mathrm{nM})$ protein concentration displayed from bottom to top. Applying a Langmuir 1:1 binding model, the signals detected (red) were fitted (black dotted lines) and rate constants for association ( $\left.k_{\text {ass }}\right)$ and dissociation ( $\left.k_{\text {diss }}\right)$ were obtained by global fit analysis. The resulting kinetic equilibrium data are summarised in Table 2. 

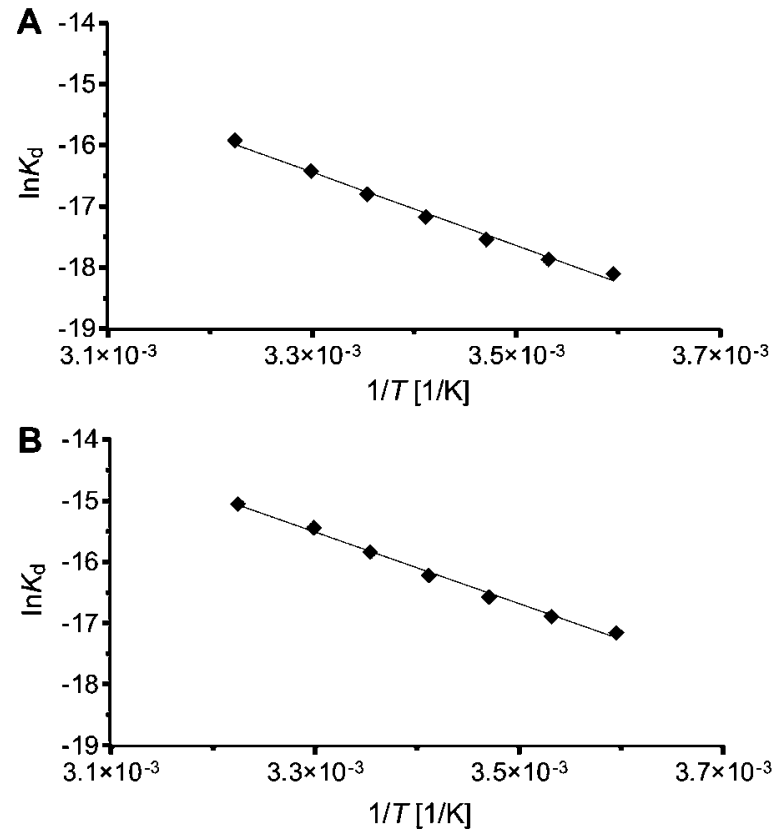

Figure 5 Equilibrium thermodynamic data analysis using the Biacore T100 system.

Equilibrium constants determined from $k_{\text {ass }}$ and $k_{\text {diss }}$ are plotted as $\ln \left(K_{\mathrm{d}}\right)$ against the reciprocal absolute temperature (van't Hoff plot) for 6-AH-CAMP (A) and 8-AHA-cAMP (B). Equilibrium thermodynamic data derived from SPR analysis are shown in Table 2.

Table 2 Equilibrium thermodynamic constants measured on the Biacore T100 system.

\begin{tabular}{lcccc}
\hline Ligand & $\begin{array}{c}K_{\mathrm{d}}{ }^{\mathrm{a}} \\
(\mathrm{nM})\end{array}$ & $\begin{array}{c}\Delta G^{\mathrm{a}} \\
\left(\mathrm{kcal} \mathrm{mol}^{-1}\right)\end{array}$ & $\begin{array}{c}\Delta H \\
\left(\mathrm{kcal} \mathrm{mol}^{-1}\right)\end{array}$ & $\begin{array}{c}\Delta S \\
\left(\mathrm{cal} \mathrm{mol}^{-1}\right. \\
\left.\mathrm{K}^{-1}\right)\end{array}$ \\
\hline 6-AH-cAMP & $38 \pm 5$ & $-9.9 \pm 0.1$ & $-11.9 \pm 0.1$ & $-7 \pm 1$ \\
8-AHA-cAMP & $110 \pm 21$ & $-9.4 \pm 0.1$ & $-12.2 \pm 0.4$ & $-10 \pm 2$ \\
\hline
\end{tabular}

a Determined at $293 \mathrm{~K}$.

sation. A favourable $\Delta H$ term implies better complementarity of the binding interfaces, because enthalpy corresponds to the energy associated with the net change in non-covalent bonds (Ladbury, 2004). Thermodynamic data also open the way for the design of more potent and selective inhibitors (Yin et al., 2006). This is exemplified by the development of second-generation HIV protease inhibitors (Ohtaka et al., 2002).

Potential biomedical applications of cAMP analogues in PKA-related research fostered the study of cyclic nucleotide binding and resulted in the synthesis of hundreds of cyclic nucleotides since the 1960s (Schwede et al., 2000b). This led to an overwhelming amount of binding data for PKA, subunits thereof and their interaction partners. In this study, we started to analyse thermodynamic aspects of the cyclic nucleotide/PKA interaction. To this end, we compared two independent methods for the determination of thermodynamic data for the interaction of a PKA R subunit and analogues of its natural binding partner CAMP. ITC is the standard method for thermodynamic analysis and directly records the heat changes during an interaction. This yielded equilibrium thermodynamic data $(\Delta H$, free energy $\Delta G$ and entropy
A
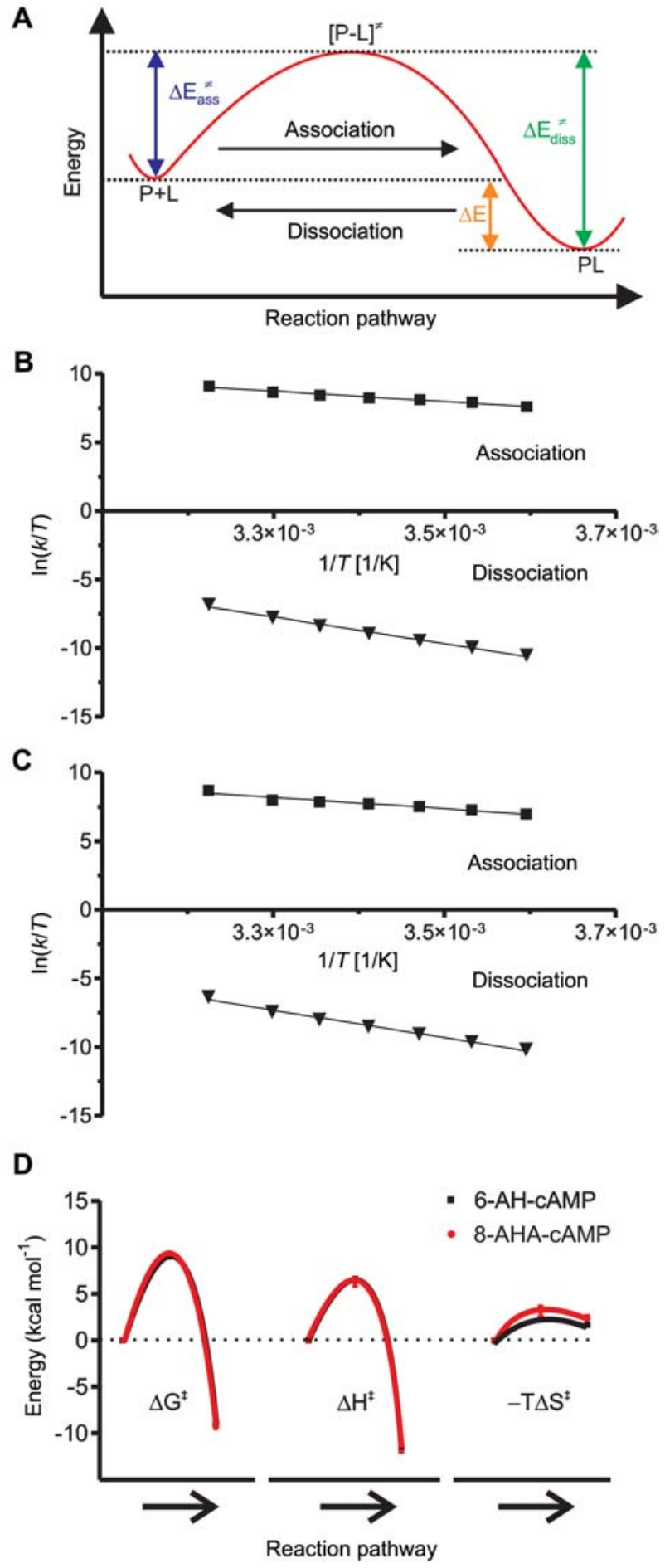

Figure 6 Transition state thermodynamics.

(A) Schematic energy diagram of a transition state. During association, the protein $(P)$ and ligand $(L)$ form an activated complex $[\mathrm{P}-\mathrm{L}]^{*}$. The energy of this transition state is elevated by $\Delta E_{\text {ass }}^{\neq}$in which $\Delta E$ can be $\Delta G, \Delta H$ or $-T \Delta S$. $[\mathrm{P}-\mathrm{L}]^{\neq}$changes into the product $\mathrm{PL}$. The energy level of $\mathrm{PL}$ is reduced by $\Delta E_{\text {ass }}^{\neq}$compared to the transition state. $\Delta E_{\text {diss }}^{*}$ also represents the activation energy barrier of the dissociation reaction. The difference in energy levels for $\mathrm{PL}$ and $\mathrm{P}+\mathrm{L}$, the equilibrium thermodynamic data, is the difference between $\Delta E_{\text {ass }}^{\neq}$and $\Delta E_{\text {diss. }}^{\neq}$. For both, association and dissociation rate constants, calculated $\ln (k / T)$ values are plotted as a function of the reciprocal absolute temperature (Eyring plot) for 6-AH-CAMP (B) and 8-AHA-CAMP (C). The resulting thermodynamic transition-state data are summarised in Table 3. (D) Energy profiles of the interactions of 6-AH-cAMP (black) and 8AHA-cAMP with the regulatory PKA subunit bRl $\alpha$ 92-260. Each energy term is depicted according to $(A)$. 
Table 3 Transition state thermodynamic constants measured on the Biacore T100 system.

\begin{tabular}{lcccccc}
\hline Ligand & $\begin{array}{c}\Delta G_{\text {ass }}^{\neq} \\
\left(\mathrm{kcal} \mathrm{mol}^{-1}\right)\end{array}$ & $\begin{array}{c}\Delta H_{\text {ass }}^{\neq} \\
\left(\mathrm{kcal} \mathrm{mol}^{-1}\right)\end{array}$ & $\begin{array}{c}\Delta S_{\text {ass }}^{\neq} \\
\left(\mathrm{cal} \mathrm{mol}^{-1} \mathrm{~K}^{-1}\right)\end{array}$ & $\begin{array}{c}\Delta G_{\text {diss }}^{\neq} \\
\left(\mathrm{kcal} \mathrm{mol}^{-1}\right)\end{array}$ & $\begin{array}{c}\Delta H_{\text {diss }}^{\neq} \\
\left(\mathrm{kcal} \mathrm{mol}^{-1}\right)\end{array}$ & $\begin{array}{c}\Delta S_{\text {diss }}^{*} \\
\left(\mathrm{cal} \mathrm{mol}^{-1} \mathrm{~K}^{-1}\right)\end{array}$ \\
\hline 6-AH-cAMP & $9.1 \pm 0.1$ & $6.6 \pm 0.1$ & $-8 \pm 1$ & $19.0 \pm 0.1$ & $18.2 \pm 0.2$ & $-3 \pm 1$ \\
8-AHA-cAMP & $9.4 \pm 0.1$ & $6.3 \pm 0.6$ & $-11 \pm 3$ & $18.8 \pm 0.1$ & $18.0 \pm 1.0$ & $-3 \pm 1.5$ \\
\hline
\end{tabular}

aDetermined at $293 \mathrm{~K}$.

$\Delta S$ ) for the interactions (Table 1), which also were obtained indirectly using kinetic analyses based on SPR (Table 2). ITC analysis showed affinity between the bovine PKA R subunit Rl $\alpha 92-260$ and cAMP $\left(K_{d}=38 \pm\right.$ $6 \mathrm{nM}$ ) that is in good agreement with data from competition experiments (Hahnefeld et al., 2005). The interaction is exothermic and only enthalpically driven. For unmodified CAMP, thermodynamic data cannot be obtained from SPR measurements (see results). It therefore was important to investigate an analogue of cAMP, 8-AHA-cAMP (Figure 2), known to have similar affinity for the PKA regulatory subunit that could also be immobilised for SPR analysis (Moll et al., 2006). Indeed, in ITC experiments, the $K_{d}$ for 8-AHA-CAMP was identical to that for cAMP (Table 1). Although the $\Delta G$ value for 8AHA-cAMP was slightly lower in the SPR analysis, the contributions of $\Delta H$ and $-T \Delta S$ to the free energy were the same as observed by ITC. This implies that coupling to the sensor surface does not change the way in which the protein interacts with 8-AHA-cAMP (Figure 7).

The ITC data for CAMP and 8-AHA-cAMP presented here reveal that, although the binding strength was iden-

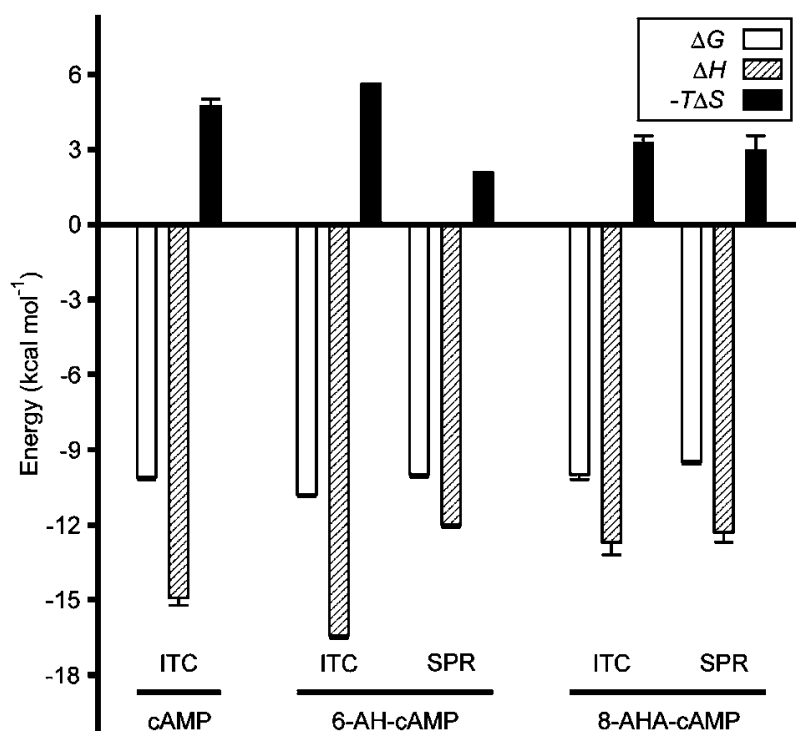

Figure 7 Comparison of thermodynamic equilibrium parameters obtained by ITC and SPR.

Data are summarised for the interaction of the regulatory PKA subunit bRl $\alpha$ 92-260 with cAMP and the two analogues $6-\mathrm{AH}-$ cAMP and 8-AHA-cAMP. All energy terms are shown in kcal mol- $^{-1}$ with free energy $(\Delta G)$ in white and its enthalpic $(\Delta H)$ and entropic $(-T \Delta S)$ contributions in hatched and black columns, respectively. The affinity $(\Delta G)$ of either analogue for the protein is similar according to both methods, but slightly reduced in the SPR measurements. Note, however, the different entropic and enthalpic contributions to $\Delta G$ for 6-AH-cAMP, but not for 8-AHAcAMP, when comparing ITC and SPR measurements. tical, surprisingly, the enthalpic and entropic contributions to the $\Delta G$ values were not. This indicates that the mode of protein binding to the two ligands differs, although they display identical affinities (Figure 7).

Binding of 6-AH-cAMP to the PKA R subunit was stronger than for CAMP, as determined by both methods (Tables 1 and 2), as was previously observed by fluorescence polarisation and SPR using another protein construct of the PKA R subunit (Moll et al., 2006). Similarly to 8-AHA-cAMP, the binding strength of 6-AH-cAMP in SPR measurements was slightly lower compared to the ITC data (Figure 7). Unlike 8-AHA-cAMP, however, comparison of data from the two methods for 6-AH-cAMP showed significant differences $\left(>4 \mathrm{kcal} \mathrm{mol}^{-1}\right)$ in the contribution of $\Delta H$ and $-T \Delta S$ to $\Delta G$. This can most probably be attributed to the immobilisation of 6-AH-cAMP in SPR experiments. The protein binding mode may be influenced by the position at which the nucleobase is immobilised to the sensor surface. This may cause differences in the degrees of freedom of the individual analogues (Figure 2). Furthermore, the crystal structure (Figure 1) of the $\mathrm{R}$ subunit in complex with cAMP suggests different accessibility of the 8 and 6 positions. Alternatively, these data could also be explained by intrinsic differences between the two methods concerning the observable binding event: ITC experiments directly monitor heat changes for all components of the system during the reaction. SPR, on the other hand, indirectly yields thermodynamic parameters by detecting mass changes that occur upon binding at different temperatures. If substantial rearrangements of water molecules occur during binding, this event may not be detectable in equilibrium thermodynamic data derived from SPR measurements (Zeder-Lutz et al., 1997).

Comparison of ITC data for the three cyclic nucleotides indicates that enthalpy-entropy compensations lead to the binding affinities observed. Entropically driven reactions are often associated with hydrophobic interactions, conformational changes and/or water and ion release. Reactions involving the formation of hydrogen bonds and/or protonation steps, on the other hand, are frequently driven by enthalpic contributions to $\Delta G$ (Feng et al., 2004). Possibly, the differences observed in enthalpy and entropy contributions of the analogues compared to CAMP are caused by the position of their linker. Compared to CAMP, a linker at position N6 would seem to cause more changes in the binding process, while a linker at C8 would reduce the changes required. In addition, large covalent modifications at position $\mathrm{C} 8$ do not weaken binding affinities (Zimmermann and Herberg, unpublished observation).

The energy profiles of the transition state, which only could be obtained by SPR and not by ITC measure- 
ments, indicate that the protein binds rather similarly to both immobilised nucleotides (Figure 6D). Differences observed in the entropy term for the two transition states are within the error of the measurement. Thus, the same thermodynamic parameters drive complex formation of the PKA R subunit with 6-AH-cAMP and 8-AHA-cAMP.

The results of our SPR and ITC analyses allow a comparison of the methods. Equilibrium thermodynamic data of similar quality can be obtained using either experimental set-up. A drawback of ITC is its notorious material consumption, which requires the availability of large quantities of recombinant protein that is soluble at high concentrations. Suitable affinities for ITC measurements are in the nano- to millimolar range (Wiseman et al., 1989; Holdgate, 2001). For in-depth characterisation of previously identified interaction partners, however, ITC offers advantages as a homogeneous method. Both interaction partners can be used in their native state and do not need to be immobilised or modified with a label. ITC allows the determination of changes in the thermodynamic parameters of all system components by direct measurement of heat changes. A further advantage is that it is not limited by the size of the interaction partners, even allowing for the determination of metal-ion binding (Hammann et al., 2001).

Compared to ITC, protein consumption was reduced 10-fold in SPR measurements. Furthermore, sensor chips can be re-used several times. In this solid phase set-up, thermodynamic data are determined indirectly and immobilisation of one of the binding partners is required. Our data, particularly for 6-AH-CAMP, indicated that this can interfere with the binding. Thermodynamic SPR measurements only reflect the binding event itself, which might reduce the complexity of the system, but also limits the information content of data. Thermodynamic data can only be obtained from SPR analyses for the temperature range in which $\Delta H$ is constant, i.e., the heat capacity of the interaction is constant. Separate determination of thermodynamic contributions to $\Delta G$ by association and dissociation, however, allows an insight into the transition state parameters that cannot be investigated by ITC.

In summary, this parallel analysis using ITC and SPR highlights method-specific advantages and drawbacks. This first thermodynamic investigation of the binding of cAMP and two of its analogues to their main target, PKA, might advance analogue design. In this iterative process, SPR would seem to be more suitable for high-throughput screening, while ITC would be more advantageous for analysis of a specific interaction.

\section{Materials and methods}

\section{Materials}

The synthetic peptide substrate Kemptide (LRRASLG) was purchased from Biosyntan GmbH (Berlin, Germany). ATP and $\mathrm{NADH}$ were obtained from Biomol GmbH (Hamburg, Germany). cAMP (adenosine-3',5'-cyclic monophosphate), 8-AHA-cAMP [8-(6-aminohexyl)aminoadenosine--3', $5^{\prime}$-cyclic monophosphate], 6-AH-cAMP [N6-(6-aminohexyl)adenosine-3',5'-cyclic monophosphate] and 6-AH-cAMP agarose were obtained from Biolog
Life Science Institute (Bremen, Germany). Fine chemicals (research grade) were purchased from Roth (Karlsruhe, Germany) or from Sigma (Deisenhofen, Germany). CM5 sensor chips (research grade), NHS ( $N$-hydroxysuccinimide), EDC [ $N$-ethyl- $N^{\prime}-$ (dimethylaminopropyl)-carbodiimide], ethanolamine- $\mathrm{HCl}$, and surfactant P20 were obtained from Biacore AB (Uppsala, Sweden).

\section{Protein expression and purification}

The cDNA for the expression of recombinant bovine Rl $\alpha$ 92-260 was a kind gift from Prof. S.S. Taylor, University of California, San Diego, USA. Bovine RI $\alpha$ 92-260 was overexpressed in $E$. coli BL21 (DE3) RIL. For SPR measurements, the protein was purified as previously described (Hahnefeld et al., 2005). For ITC measurements, completely nucleotide-free $R$ subunit was obtained. For this purpose, protein was eluted from 6-AH-cAMP agarose with $8 \mathrm{M}$ urea, $20 \mathrm{~mm}$ MOPS $(\mathrm{pH} 7.0)$ at room temperature. The protein was extensively dialysed against $150 \mathrm{~mm}$ $\mathrm{NaCl}, 20 \mathrm{~mm}$ MOPS, $10 \mathrm{~mm} \mathrm{MgCl}$, 1 mm ATP, pH 7.0 (buffer A). The purity of the $R$ subunit was confirmed by SDS-PAGE (Laemmli, 1970) and the biological activity of the protein was verified using the phosphotransferase assay with the peptide Kemptide (LRRASLG) as a substrate, as previously described (Cook et al., 1982). Protein concentration was measured by colorimetric assay using BSA as a standard (Bradford, 1976).

\section{ITC analysis}

The interaction between the $\mathrm{R}$ subunit and cAMP, 8-AHA-cAMP or 6-AH-cAMP was analysed in buffer A supplemented with $1 \mathrm{~mm} \beta$-mercaptoethanol using a VP-ITC microcalorimeter (MicroCal LLC, Northampton, MA, USA). The R subunit (10 $\mu \mathrm{M})$ was equilibrated in the $1.4-\mathrm{ml}$ sample cell at $20.0^{\circ} \mathrm{C}$. The nucleotide concentration in the syringe was $271 \mu \mathrm{M}$ (cAMP) or $140 \mu \mathrm{M}$ (analogues). To ensure that the nucleotide concentration was at its loading value, two injections ( $1 \mu$ l each) were performed before the actual titration experiment (Mizoue and Tellinghuisen, 2004). Nucleotide was injected (cAMP, $5 \mu \mathrm{l} ; 6-\mathrm{AH}-$ cAMP and 8-AHA-cAMP, $10 \mu \mathrm{l}$ ) into the pre-equilibrated PKA R subunit and heat changes were recorded. After the heat signal had returned to baseline level (approx. $4 \mathrm{~min}$ ), a new injection was started. To minimise artefacts, nucleotides were dissolved in the dialysis buffer used for protein purification. For blank subtractions, nucleotides were injected into buffer only using identical steps. Data evaluation was performed with the software MicroCal Origin for ITC (MicroCal LLC; Wiseman et al., 1989), including corrections for volume changes during the titration. The correct protein concentration in the cell was calculated from the ITC experiment with a stoichiometry of $n=1$. Every experiment was performed at least in duplicate and errors are reported as standard deviation.

\section{Surface plasmon resonance analysis}

All SPR interaction analyses were performed in buffer A plus $0.005 \%$ (v/v) surfactant P20 using a Biacore T100 instrument. For covalent coupling of 8-AHA-cAMP and 6AH-cAMP, carboxymethylated sensor chip surfaces (CM5, research grade) were activated with NHS/EDC for $7 \mathrm{~min}$ and CAMP analogue (35 $\mu \mathrm{M}$ in $100 \mathrm{~mm}$ borate buffer, $\mathrm{pH}$ 8.5) was injected for $7 \mathrm{~min}$ at a flow rate of $5 \mu \mathrm{l} / \mathrm{min}$. Deactivation of the surface was performed using $1 \mathrm{M}$ ethanolamine- $\mathrm{HCl}(\mathrm{pH}$ 8.5) for $7 \mathrm{~min}$. Reference cells (flow cells 1 and 3 ) were activated accordingly and deactivated subsequently. Kinetic analyses were performed by injection of increasing concentrations $(2.5,5,10,20,40,80,160$ (in duplicate), 320 and $640 \mathrm{~nm}$, and $1.28 \mu \mathrm{M}$ ) of the $\mathrm{R}$ subunit at a flow rate of $30 \mu \mathrm{l} / \mathrm{min}$. For each temperature $\left(5^{\circ} \mathrm{C}, 10^{\circ} \mathrm{C}\right.$, 
$15^{\circ} \mathrm{C}, 20^{\circ} \mathrm{C}, 25^{\circ} \mathrm{C}, 30^{\circ} \mathrm{C}$ and $37^{\circ} \mathrm{C}$ ), association and dissociation signals were each monitored for $3 \mathrm{~min}$. The sensor surfaces were regenerated after each binding cycle by two injections of $3 \mathrm{~m}$ guanidinium $\mathrm{HCl}$. After subtracting the reference cell signal, resulting binding signals were fitted. Data evaluation was performed using the Biacore T100 evaluation software, version 1.0.

\section{Acknowledgements}

We thank Katja Eildermann and Katrin Guske for superb technical help, Prof. Susan Taylor (University of San Diego, USA) for providing vector DNA, and Dr. Frank Schwede and Dr. HansGottfried Genieser (Biolog, Bremen) for cyclic nucleotide analogues. Biacore $A B$ is acknowledged for providing access to a Biacore T100 system. This work was supported by grants from Deutsche Forschungsgemeinschaft (DFG, He1818/4 and HA3459/3) to F.W. Herberg and C. Hammann and from BMBF (01 GR 0441, NGFN) to F.W. Herberg. Sonja Schweinsberg was supported by the University of Kassel graduate program.

\section{References}

Aandahl, E.M., Aukrust, P., Skalhegg, B.S., Muller, F., Froland, S.S., Hansson, V., and Tasken, K. (1998). Protein kinase A type I antagonist restores immune responses of $T$ cells from HIV-infected patients. FASEB J. 12, 855-862.

Anand, G.S., Law, D., Mandell, J.G., Snead, A.N., Tsigelny, I., Taylor, S.S., Eyck, L.F., and Komives, E.A. (2003). Identification of the protein kinase A regulatory Rl $\alpha$-catalytic subunit interface by amide $\mathrm{H} / 2 \mathrm{H}$ exchange and protein docking. Proc. Natl. Acad. Sci. USA 100, 13264-13269.

Berman, H.M., Ten Eyck, L.F., Goodsell, D.S., Haste, N.M., Kornev, A., and Taylor, S.S. (2005). The cAMP binding domain: an ancient signaling module. Proc. Natl. Acad. Sci. USA 102, 45-50.

Bradford, M.M. (1976). A rapid and sensitive method for the quantitation of microgram quantities of protein utilizing the principle of protein-dye binding. Anal. Biochem. 72, 248254

Cook, P.F., Neville, M.E. Jr., Vrana, K.E., Hartl, F.T., and Roskoski, R. Jr. (1982). Adenosine cyclic 3',5'-monophosphate dependent protein kinase: kinetic mechanism for the bovine skeletal muscle catalytic subunit. Biochemistry 21, 5794-5799.

Doyle, M.L., Myszka, D.G., and Chaiken, I.M. (1996). Molecular interaction analysis in ligand design using mass transport, kinetic and thermodynamic methods. J. Mol. Recognit. 9, 65-74.

Ek, I., Arner, P., Ryden, M., Holm, C., Thorne, A., Hoffstedt, J., and Wahrenberg, H. (2002). A unique defect in the regulation of visceral fat cell lipolysis in the polycystic ovary syndrome as an early link to insulin resistance. Diabetes $51,484-492$.

Feng, M., Patel, D., Dervan, J.J., Ceska, T., Suck, D., Haq, I., and Sayers, J.R. (2004). Roles of divalent metal ions in flap endonuclease-substrate interactions. Nat. Struct. Mol. Biol. $11,450-456$.

Gesellchen, F., Zimmermann, B., and Herberg, F.W. (2005). Direct optical detection of protein-ligand interactions. Methods Mol. Biol. 305, 17-46.

Hahnefeld, C., Drewianka, S., and Herberg, F.W. (2004). Determination of kinetic data using surface plasmon resonance biosensors. Methods Mol. Med. 94, 299-320.

Hahnefeld, C., Moll, D., Goette, M., and Herberg, F.W. (2005). Rearrangements in a hydrophobic core region mediate cAMP action in the regulatory subunit of PKA. Biol. Chem. 386, 623-631.
Hammann, C., Cooper, A., and Lilley, D.M. (2001). Thermodynamics of ion-induced RNA folding in the hammerhead ribozyme: an isothermal titration calorimetric study. Biochemistry 40, 1423-1429.

Herberg, F.W. and Zimmermann, B. (1999). Analysis of protein kinase interactions using biomolecular interaction analysis. In: Protein Phosphorylation - A Practical Approach, D.G. Hardie, ed. (Oxford, UK: Oxford University Press), pp. 335371.

Holdgate, G.A. (2001). Making cool drugs hot: isothermal titration calorimetry as a tool to study binding energetics. Biotechniques $31,164-166,168,170$ passim.

Holdgate, G.A. and Ward, W.H.J. (2005). Measurements of binding thermodynamics in drug discovery. Drug Discov. Today 10, 1543-1550.

Johnsson, B., Lofas, S., and Lindquist, G. (1991). Immobilization of proteins to a carboxymethyldextran-modified gold surface for biospecific interaction analysis in surface plasmon resonance sensors. Anal. Biochem. 198, 268-277.

Karlsson, R. and Falt, A. (1997). Experimental design for kinetic analysis of protein-protein interactions with surface plasmon resonance biosensors. J. Immunol. Methods 200, 121-133.

Kim, C., Xuong, N.H., and Taylor, S.S. (2005). Crystal structure of a complex between the catalytic and regulatory $(\mathrm{Rl} \alpha)$ subunits of PKA. Science 307, 690-696.

Ladbury, J.E. (2004). Application of isothermal titration calorimetry in the biological sciences: things are heating up! Biotechniques $37,885-887$.

Ladbury, J.E. and Chowdhry, B.Z. (1996). Sensing the heat: the application of isothermal titration calorimetry to thermodynamic studies of biomolecular interactions. Chem. Biol. 3, 791-801.

Laemmli, U.K. (1970). Cleavage of structural proteins during assembly of the head of bacteriophage T4. Nature 227, 680-685.

Langmuir, I. (1916). The constitution and fundamental properties of solids and liquids. Part I. Solids. J. Am. Chem. Soc. 38, 2221-2295.

Langmuir, I. (1918). The adsorption of gases on plane surfaces of glass, mica and platinum. J. Am. Chem. Soc. 40, 13611404.

Manning, G., Whyte, D.B., Martinez, R., Hunter, T., and Sudarsanam, S. (2002). The protein kinase complement of the human genome. Science 298, 1912-1934.

Mizoue, L.S. and Tellinghuisen, J. (2004). The role of backlash in the 'first injection anomaly' in isothermal titration calorimetry. Anal. Biochem. 326, 125-127.

Moll, D., Prinz, A., Gesellchen, F., Drewianka, S., Zimmermann, B., and Herberg, F.W. (2006). Biomolecular interaction analysis in functional proteomics. J. Neural Transm. 113, 10151032.

Noble, M.E., Endicott, J.A., and Johnson, L.N. (2004). Protein kinase inhibitors: insights into drug design from structure. Science 303, 1800-1805.

Ogreid, D., Ekanger, R., Suva, R.H., Miller, J.P., and Doskeland, S.O. (1989). Comparison of the two classes of binding sites (A and B) of type I and type II cyclic-AMP-dependent protein kinases by using cyclic nucleotide analogs. Eur. J. Biochem. 181, 19-31.

Ohtaka, H., Velazquez-Campoy, A., Xie, D., and Freire, E. (2002). Overcoming drug resistance in HIV-1 chemotherapy: the binding thermodynamics of Amprenavir and TMC-126 to wild-type and drug-resistant mutants of the HIV-1 protease. Protein Sci. 11, 1908-1916.

Rahmouni, S., Aandahl, E.M., Trebak, M., Boniver, J., Tasken, K., and Moutschen, M. (2001). Increased cAMP levels and protein kinase (PKA) type I activation in CD4 ${ }^{+} \mathrm{T}$ cells and $\mathrm{B}$ cells contribute to retrovirus-induced immunodeficiency of mice (MAIDS): a useful in vivo model for drug testing. FASEB J. $15,1466-1468$. 
Ringheim, G.E. and Taylor, S.S. (1990). Dissecting the domain structure of the regulatory subunit of cAMP-dependent protein kinase I and elucidating the role of MgATP. J. Biol. Chem. 265, 4800-4808.

Schwede, F., Christensen, A., Liauw, S., Hippe, T., Kopperud, R., Jastorff, B., and Doskeland, S.O. (2000a). 8-Substituted CAMP analogues reveal marked differences in adaptability, hydrogen bonding, and charge accommodation between homologous binding sites (Al/All and $\mathrm{BI} / \mathrm{BII}$ ) in cAMP kinase I and II. Biochemistry 39, 8803-8812.

Schwede, F., Maronde, E., Genieser, H., and Jastorff, B. (2000b). Cyclic nucleotide analogs as biochemical tools and prospective drugs. Pharmacol. Ther. 87, 199-226.

Stergiopoulos, S.G. and Stratakis, C.A. (2003). Human tumors associated with Carney complex and germline PRKAR1A mutations: a protein kinase A disease! FEBS Lett. 546, 5964.

Stratakis, C.A. and Cho-Chung, Y.S. (2002). Protein kinase A and human disease. Trends Endocrinol. Metab. 13, 50-52.

Su, Y., Dostmann, W.R., Herberg, F.W., Durick, K., Xuong, N.H., Ten Eyck, L., Taylor, S.S., and Varughese, K.I. (1995). Regulatory subunit of protein kinase $A$ : structure of deletion mutant with cAMP binding domains. Science 269, 807-813.

Whitesides, G.M. and Krishnamurthy, V.M. (2006). Designing ligands to bind proteins. Q. Rev. Biophys. 38, 385-395.
Wiseman, T., Williston, S., Brandts, J.F., and Lin, L.N. (1989). Rapid measurement of binding constants and heats of binding using a new titration calorimeter. Anal. Biochem. 179, 131-137.

Wu, J., Brown, S., Xuong, N.H., and Taylor, S.S. (2004a). Rl $\alpha$ subunit of PKA; a cAMP-free structure reveals a hydrophobic capping mechanism for docking cAMP into site B. Structure (Camb.) 12, 1056-1064.

Wu, J., Jones, J.M., Xuong, N.H., Eyck, L.F., and Taylor, S.S. (2004b). Crystal structures of Rl $\alpha$ subunit of cyclic adenosine $5^{\prime}$-monophosphate (cAMP)-dependent protein kinase complexed with $[\mathrm{R}(\mathrm{p})]$-adenosine $3^{\prime}, 5^{\prime}$-cyclic monophosphothioate and $[S(p)]$-adenosine $3^{\prime}, 5^{\prime}$-cyclic monophosphothioate, the phosphothioate analogues of cAMP. Biochemistry $43,6620-6629$.

Yin, F., Cao, R., Goddard, A., Zhang, Y., and Oldfield, E. (2006). Enthalpy versus entropy-driven binding of bisphosphonates to farnesyl diphosphate synthase. J. Am. Chem. Soc. 128, 3524-3525.

Zeder-Lutz, G., Zuber, E., Witz, J., and Van Regenmortel, M.H. (1997). Thermodynamic analysis of antigen-antibody binding using biosensor measurements at different temperatures. Anal. Biochem. 246, 123-132.

Received August 29, 2006; accepted October 20, 2006 